\title{
Sex-Based Differences in Recurrence Rates Following Arthroscopic Anterior Shoulder Stabilization: A Systematic Review
}

\author{
Connor K. Cannizzaro, B.S., Hayden B. Schuette, D.O., Darby A. Houck, B.A., \\ Michelle L. Wolcott, M.D., Armando F. Vidal, M.D., Eric C. McCarty, M.D., \\ Jonathan T. Bravman, M.D., and Rachel M. Frank, M.D.
}

\begin{abstract}
Purpose: The purpose of this systematic review was to examine the effect that sex has on recurrence of anterior shoulder instability following primary arthroscopic anterior shoulder stabilization. Methods: A systematic review using PRISMA guidelines was performed by searching PubMed, Embase and Cochrane Library databases to identify studies reporting recurrence rates following arthroscopic anterior shoulder stabilization with a minimum follow-up period of 2 years. Patient demographics as well as preoperative, intraoperative and postoperative findings, including patient-reported outcomes and recurrence rates, were analyzed by 2 independent reviewers. Recurrence was defined as an episode of dislocation, subluxation or instability following primary arthroscopic anterior shoulder stabilization. Study methodological quality was evaluated with the Modified Coleman Methodology Score (MCMS). Risk bias was evaluated using the Methodological Index for Non-randomized Studies (MINORS score). Descriptive statistics are presented. Results: Ten studies (1 Level I, 1 Level II, 5 Level III, and 3 Level IV), including a total of 7,102 patients with primary traumatic and/or recurrent traumatic shoulder instability without previous procedures who underwent primary arthroscopic anterior shoulder stabilization, were included (mean follow-up, 34.6 months; mean age, 25.4 years). There was a total of 5,097 males $(71.8 \%)$ and 2,005 females $(28.2 \%)$. There was a higher recurrence rate in males $(6 \%-37 \%)$ compared to females $(0-32 \%)$. Clinical outcomes were inconsistently reported, so no quantitative analysis of clinical outcomes or return-tosport between sexes was possible. The average MCMS of all 10 studies was $76.8 \pm 8.0$, indicating good methodology. Conclusions: Males may have higher recurrence rates than females following primary arthroscopic anterior shoulder stabilization; however, the heterogeneity of the included studies precludes any definitive conclusions. Level of evidence: IV, Systematic Review of Level I-IV.
\end{abstract}

$\mathbf{R}$ ecurrent anterior shoulder instability is a common diagnosis in young and active patients. ${ }^{1-4}$ For appropriately indicated patients, surgical management is reliable and effective, particularly for young and active patients who are at the highest risk of developing recurrent instability without surgical management. Arthroscopic stabilization aids in restoring the attachment of the capsulolabral ligaments to the anterior

From the University of Colorado School of Medicine, Department of Orthopedics, Aurora, CO (C.K.C., D.A.H., M.L.W., A.F.V., E.C.M., J.T.B., R.M.F.) and OhioHealth, Department of Orthopedics, Columbus, $\mathrm{OH}$ (H.B.S.).

The above authors have all contributed substantial contributions to the conception or design of the work or the acquisition, analysis or interpretation of data for the work and drafting the work or revising it critically for important intellectual content, and final approval of the version to be published, as well as agreement to be accountable for all aspects of the work in ensuring that questions related to the accuracy or integrity of any part of the work are appropriately investigated and resolved. Full ICMJE author glenoid rim and generally allows for favorable outcomes in eliminating glenohumeral instability. ${ }^{5,6}$ Unfortunately, following surgical stabilization surgery, recurrence, often defined as a recurrent dislocation or subluxation, ${ }^{7,8}$ is not uncommon, with recurrence rates reported to be between 0 and $37.5 \% .^{9}$ Risk factors for recurrent instability following arthroscopic labral repair include participation in contact sports, age, sex,

\footnotetext{
disclosure forms are available for this article online, as supplementary material.

Received August 6, 2019; accepted April 8, 2020.

Address correspondence to Rachel M. Frank, M.D., 2150 Stadium Drive, Room 222, Boulder, CO 80309, U.S.A. E-mail:Rachel.Frank@cuanschutz. edu

(C) 2020 THE AUTHORS. Published by Elsevier Inc. on behalf of the Arthroscopy Association of North America. This is an open access article under the CC BY-NC-ND license (http://creativecommons.org/licenses/by-nc-nd/4.0/). 2666-061X/19861

https://doi.org/10.1016/j.asmr.2020.04.004
} 
technical error, number of dislocations prior to surgery, hyperlaxity, and bone loss on the glenoid and/or humeral head. ${ }^{10,11}$ Multiple studies have reported that male sex is a risk factor of recurrence ${ }^{8,10}$; however, other studies have disputed that claim. ${ }^{7,12}$ The purpose of this systematic review was to examine the effect that sex has on recurrence of anterior shoulder instability following primary arthroscopic anterior shoulder stabilization. It was hypothesized that males would have higher recurrence rates than females following arthroscopic anterior shoulder stabilization.

\section{Methods}

This systematic review was performed by following the Preferred Reporting Items for Systematics Reviews and Meta-Analyses (PRISMA) guidelines. Two independent reviewers (CKC, HBS) searched PubMed, the Cochrane Library and Embase Library through May 25, 2018. The following search terms were used: "shoulder AND repair AND anterior AND (recurrence OR recurrent) AND (instability OR dislocation OR subluxation) AND (arthroscopic OR arthroscopy) NOT open NOT acromioclavicular." Exclusion and inclusion criteria were established prior to the search and were used to identify the included studies. After duplicates were identified, a total of 399 studies were accessed for inclusion based on title and/or abstract. The inclusion criteria consisted of studies comparing failure or recurrence rates between males and females following primary (first attempt at surgical management) arthroscopic anterior shoulder stabilization and studies with a minimum follow-up of 24 months. Exclusion criteria consisted of revision surgery studies, open surgery studies, non-English studies, case reports studies, reviews and/or meta-analyses studies, perception-based studies, scientific meetings and/or abstract proceedings, biomechanical studies, novel technique studies, current concept studies, and Level V evidence studies. Data extraction was completed separately by each independent reviewer and then compared. Discrepancies in data were identified, discussed and resolved by a third independent researcher (a professional research assistant in Orthopedics) (DAH).

\section{Data Extraction}

Demographic data, including patient's age at the time of surgery, patient's sex and follow-up time, were collected. Recurrence rates were extracted from each study. Additionally, each study was further evaluated to see whether other clinical outcome measures were used when comparing male to female outcomes.

\section{Study Methodology Assessment}

The Modified Coleman Methodology Score (MCMS) ${ }^{13}$ was used to access the quality of the studies' methodologies. MCMS is based on a scale ranging from
0 -100. Scores ranging between 85 and 100 are excellent, between 70 and 84 are good, between 55 and 69 are fair, and a score of less than 55 is considered poor. The Methodological Index for Nonrandomized Studies (MINORS) score was used to evaluate the risk bias of the included studies. The MINORS score is based on 8 criteria that are scored on a scale of 0 to 2; a score of 2 indicates that the criteria were reported adequately; 1 indicates that it was reported but inadequately; and a score of 0 indicates that it was not reported. ${ }^{14}$ An overall score of 16 is considered a perfect score and indicates a low risk of bias. As the MINORS score decreases, the risk of bias increases accordingly. ${ }^{14}$

\section{Statistical Analysis}

Because of the nature of the included studies, their high heterogeneity and their inconsistent methodologies, pooling of data and weighted mean calculations were avoided. Instead, a subjective synthesis was performed, in which ranges were reported, and the data from each individual study were presented in a forest plot. For dichotomous data, a random-effects model (due to anticipated heterogeneity) was used by the Mantel-Haenszel method to calculate risk ratios with 95\% confidence intervals (CI) for individual study results and was presented in a forest plot. According to Cote et al., ${ }^{15}$ pooling of low-evidence data should be avoided because it carries a high risk of bias, and possible confounding variables are not able to be accounted for. Therefore, summary estimates were avoided when presenting data in the forest plot. In addition, Cote et al. ${ }^{15}$ reported that exploration heterogeneity in a systematic review is valuable for examining the strengths and weakness of the current literature, which can be of use to direct future highquality research. ${ }^{15}$ Thus, the $I^{2}$ statistic was calculated in order to quantify the degree of heterogeneity. In addition, we accounted for study design to test whether the recurrence rates differed by the Level of Evidence of the study. Forest plots were created using Review Manager (RevMan) software, version 5.3.5, The Cochrane Collaboration, London, United Kingdom). ${ }^{16}$

Ten studies ${ }^{7,8,10,17-23}$ met inclusion and exclusion criteria (1 Level I, 1 Level II, 5 Level III, and 3 Level IV) (Fig 1). The main reasons for exclusion were; $\leq 24$-month follow-up, novel surgical techniques, case report studies, reviews and meta-analyses, and Level V evidence research.

\section{Patient Demographics}

A total of 7,097 patients were included in this systematic review: 5,092 males (71.7\%) and 2,005 females $(28.3 \%)$ (Table 1$)$. It is important to note that $81 \%$ of the patients who were included were from 1 study, 
Fig 1. Preferred Reporting Items for Systematics Reviews and Meta-Analyses (PRISMA) Flow Diagram.

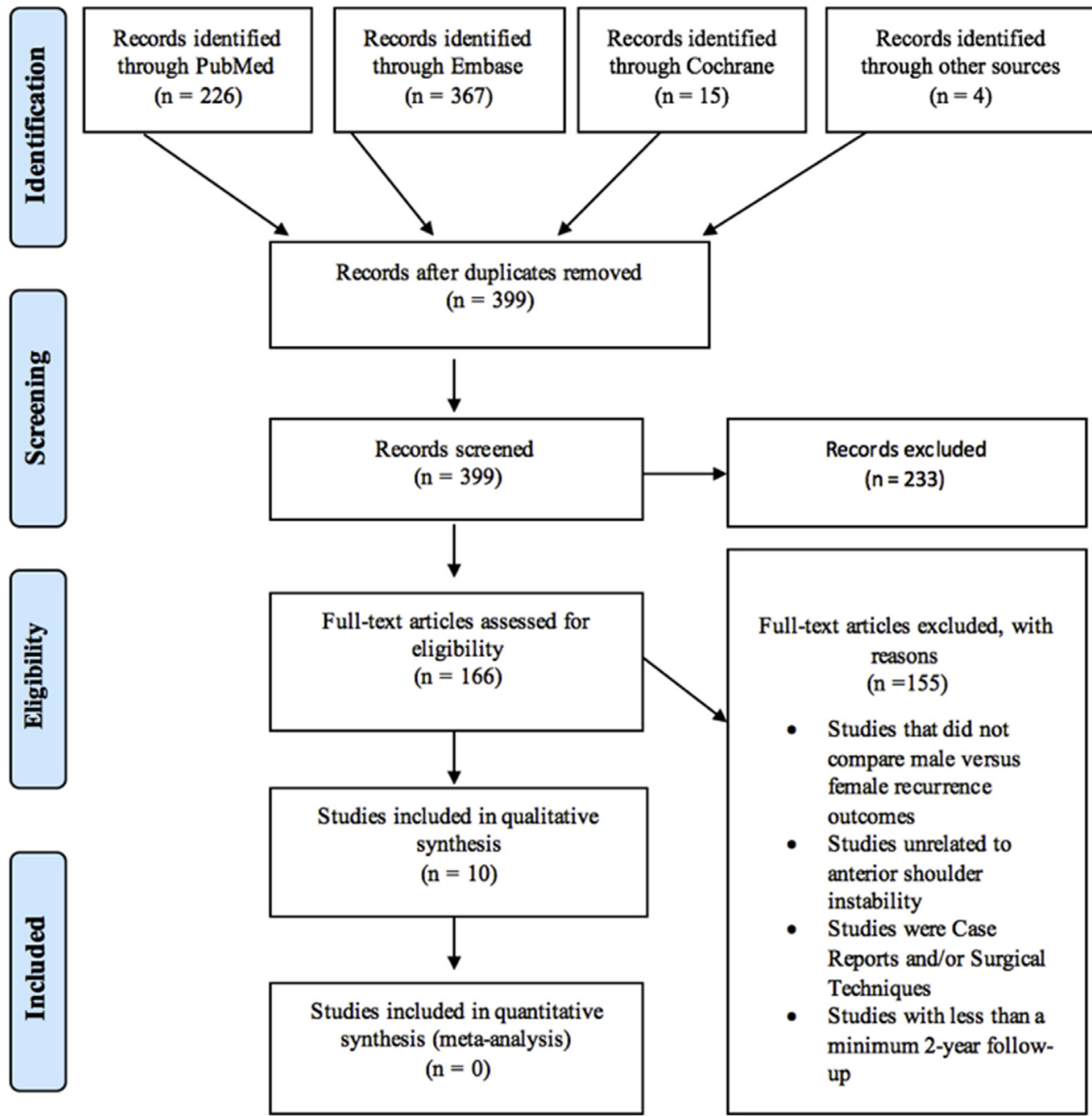

Mahure et al. ${ }^{22}$ The age of the patients at the times of surgery ranged from 14 years to 69 years of age. All studies had a minimum follow-up of 24 months (range 24-120 months).

\section{Procedures Performed}

All studies performed arthroscopic Bankart repair. In addition, $8^{7,8,12,17-20,23}$ of the 10 studies performed other procedures at the time of the arthroscopic Bankart repair, whereas 2 studies did not report performing other procedures. $^{10,22}$ Ahmed et al. ${ }^{8}$ performed Bankart repair with capsular shift in all patients. Boileau et al. ${ }^{17}$ repaired superior labral anterior to posterior tear (SLAP) lesions if present at the time of surgery. In 1 study, by Chen et al., ${ }^{20}$ the patients were separated into 2 groups: those undergoing isolated Bankart repair versus those undergoing Bankart repair accompanied by thermal capsular shrinkage; only the patients who underwent Bankart repair without thermal capsular shrinkage were included in this study. ${ }^{20}$ One patient in the Chen et al. ${ }^{20}$ study was identified to have a SLAP lesion that was repaired at the time of Bankart repair. Almeida Filho et al. ${ }^{12}$ performed additional capsuloplasty when indicated; however, a breakdown of patients who underwent the capsuloplasty was not reported. Flinkkila et al. ${ }^{18}$ performed additional SLAP lesion repairs in 22 patients, posterior labrum repair in 4 patients, rotator cuff repair in 2 patients, and repair of the supraspinatus in 2 patients. Nakagawa et al. ${ }^{23}$ performed concomitant capsular lesion repairs in 37 patients, as well as the repair of humeral avulsion glenohumeral ligament repairs in 2 patients. Thal et al. ${ }^{7}$ performed SLAP lesion repairs in 20 patients, repair of superior labral tears in 2 patients, repair of a supraspinatus tear in 1 patient, as well as in 1 patient with distal clavicle extension. Van der Linde et al. ${ }^{19}$ performed capsular plication if the capsule was identified to be stretched out; the number of patients to undergo capsular plication was not reported.

Eight $^{7,10,12,17-20,23}$ of the 10 studies clearly reported the patient position used in the procedure, whereas 2 studies $^{8,22}$ did not. Of the 8 studies that did report 
Table 1. Characteristics of the Included Studies

\begin{tabular}{|c|c|c|c|c|c|c|}
\hline Study & $\begin{array}{c}\text { Level of } \\
\text { Evidence }\end{array}$ & $\begin{array}{c}\text { No. of } \\
\text { Patients }\end{array}$ & $\begin{array}{c}\text { Patient age, } \\
\text { years }\end{array}$ & $\begin{array}{c}\text { Follow-up, } \\
\text { months }\end{array}$ & Men, n (\%) & Women, n (\%) \\
\hline Ahmed et al., $2012^{8}$ & $\mathrm{I}$ & 302 & $26.5(16-51)$ & $68.5(-)$ & $265(88)$ & $37(12)$ \\
\hline Boileau et al., $2007^{17}$ & III & 131 & $27.3(14-62)$ & $31.2(24-52)$ & $103(79)$ & $28(21)$ \\
\hline Chen et al., $2005^{20}$ & III & 28 & $25.6(15-44)$ & $58(30-87)$ & $20(71)$ & $8(29)$ \\
\hline Almeida Filho et al., $2012^{21}$ & III & 49 & $30(-)$ & $42.7(-)$ & $42(87)$ & $7(13)$ \\
\hline Flinkkila et al., $2010^{18}$ & IV & 174 & $27.5(15-58)$ & $51(24-95)$ & $125(72)$ & $49(28)$ \\
\hline Mahure et al., $2018^{22}$ & III & 5719 & $24.9(-)$ & $>36$ & $4013(70)$ & $1706(30)$ \\
\hline Nakagawa et al., $2017^{23}$ & III & 172 & $23.3(14-69)$ & $27.6(24-67)$ & $146(85)$ & $26(15)$ \\
\hline Porcellini et al., $2009^{10}$ & II & 385 & $28.7(16-63)$ & $>24$ & $278(72)$ & $107(28)$ \\
\hline Thal et al., $2007^{7}$ & IV & 72 & $26.7(15-64)$ & $>24(24-84)$ & $57(79)$ & $15(21)$ \\
\hline Van der Linde et al., $2011^{19}$ & IV & 65 & $31(19-56)$ & $108(96-120)$ & $43(66)$ & $22(33)$ \\
\hline
\end{tabular}

Patients' ages and follow-up times are reported as mean (range). NR, not reported.

patient positioning, 3 studies ${ }^{17,18,20}$ were performed with the patients in the beach-chair position, whereas 5 studies $^{7,10,12,19,23}$ were performed with the patient in the lateral decubitus position. Almeida Filho et al. ${ }^{12}$ described the lateral decubitus positioning with the operative limb in $50^{\circ}$ of abduction and $15^{\circ}$ of flexion. Thal et al. ${ }^{7}$ described the lateral decubitus positioning with the operative limb in $90^{\circ}$ of abduction. Porcellini et al. ${ }^{10}$ described the lateral decubitus positioning with the operative limb in $75^{\circ}$ of abduction and $25^{\circ}$ of flexion. No other studies specifically elaborated on the angling of the shoulder.

Four studies ${ }^{10,12,19,20}$ reported the portal viewing used for arthroscopy, whereas 6 studies did not. The 4 studies $^{10,12,19,20}$ reported using 3 portal views: 1 posterior portal and 2 anterior portals.

Of the studies, 2 clearly specified the operative side, whereas 8 studies did not. Boileau et al. ${ }^{17}$ included 73 right shoulders and 58 left shoulders in their study, and Flinkkila et al. ${ }^{18}$ included 119 right and 67 left shoulders in their study.

All 10 studies $^{7,8,10,17-23}$ lacked detailed reporting of magnetic resonance imaging, whether or not it was used and/or the orientation of the magnetic resonance imaging performed.

\section{Methodology Assessment}

Table 2 shows the MCMS scores from the 10 included studies. Of the 10 studies, 4 achieved excellent scores, ${ }^{7,10} 4$ achieved good scores, ${ }^{20,21,23}$ and 2 achieved fair scores. ${ }^{8,22}$ Based on the MCMS scoring scale, the overall methodology of the 10 studies included in this systematic review are good. Table 2 shows the MINORS scores for the included studies. The overall mean average score for the 10 included studies was 13.1, with scores ranging from $10^{23}$ to $15 .^{7,19}$

\section{Recurrences}

Nine studies ${ }^{7,8,10,17-21,23}$ defined recurrence as a subsequent episode of dislocation or subluxation following the initial stability procedure. One study ${ }^{22}$ defined recurrence as the need for a subsequent shoulder stabilization procedure. The overall recurrence rate ranged from $6 \%{ }^{23}$ to $35 \%$ (Table 3). ${ }^{19}$ For both prospective and retrospective studies, the recurrence rate in females ranged from $0^{7,21}$ to $37.5 \%,{ }^{20}$ whereas the recurrence rate in males ranged from $6.2 \%{ }^{23}$ to $37.2 \%{ }^{19}\left(I^{2}=0 \%\right.$ ) (Fig 2). For prospective studies, the recurrence rate in females ranged from $2.8 \%{ }^{10}$ to $32 \%,{ }^{19}$ whereas the recurrence rate in males ranged from $8.5 \%{ }^{22}$ to $37.2 \%{ }^{19}\left(I^{2}=27 \%\right)$. For retrospective studies, the recurrence rate in females ranged from $0^{7,8}$ to $37.5 \%,{ }^{20}$ whereas the recurrence rate in males ranged from $6.2 \%{ }^{23}$ to $21.6 \%{ }^{18}\left(I^{2}=0 \%\right)$.

Of the 10 studies included, 7 of the $10^{7,8,10,12,17-19}$ reported additional outcomes, whereas $3^{20,22,23}$ studies did not. Although they were reported, these secondary outcome measurements were not compared between males and females in any of the 10 included studies. Boileau et al. ${ }^{17}$ reported on shoulder range of motion, Walch-Duplay Instabilty score and Rowe score as additional outcomes to recurrence. Ahmed et al. ${ }^{8}$ reported the Western Ontario Shoulder Instability index and the Disabilties of the Arm, Shoulder and Hand scores. Almeida Filho et al. ${ }^{12}$ reported the Rowe score and the Samilson and Prieto score for shoulder joint degeneration. Flinkkila et al. ${ }^{18}$ reported the Oxford instability score and the subjective shoulder value. Thal et al. $^{7}$ reported on positive apprehension tests, American Shoulder and Elbow Surgeons Assessment Form score, Rowe score, as well as shoulder range of motion. Van der Linde et al. ${ }^{19}$ reported the Oxford instability score, Western Ontario Shoulder Instability Index and the Simple Shoulder Test as secondary outcomes along with recurrence.

Of the 10 studies, only 3 studies $^{18,21,22}$ clearly identified the age makeup of the male and female patients in their studies. Flinkkila et al. ${ }^{18}$ reported the mean age of the male and female patients to be 28 years and 26 years, respectively, and found that young age (mean age, 26 years) was statistically significantly associated with recurrence. Mahure et al. ${ }^{22}$ reported that the 
Table 2. Methodology Assessments

\begin{tabular}{|c|c|}
\hline \multicolumn{2}{|c|}{ The Modified Coleman Methodology Score (MCMS) } \\
\hline Study & MCMS \\
\hline Ahmed et al., $2012^{8}$ & 68 \\
\hline Boileau et al., $2007^{17}$ & 85 \\
\hline Chen et al., $2005^{20}$ & 70 \\
\hline Almeida Filho et al., $2012^{21}$ & 77 \\
\hline Flinkkila et al., $2010^{18}$ & 71 \\
\hline Mahure et al., $2018^{22}$ & 65 \\
\hline Nakagawa et al., $2017^{23}$ & 74 \\
\hline Porcellini et al., $2009^{10}$ & 88 \\
\hline Thal et al., $2007^{7}$ & 85 \\
\hline Van der Linde et al., $2011^{19}$ & 85 \\
\hline \multicolumn{2}{|c|}{ Methodological Index for Nonrandomized Studies (MINORS Score } \\
\hline Study & Overall Score \\
\hline Ahmed et al., $2012^{8}$ & 13 \\
\hline Boileau et al., $2007^{17}$ & 14 \\
\hline Chen et al., $2005^{20}$ & 13 \\
\hline Almeida Filho et al., $2012^{21}$ & 11 \\
\hline Flinkkila et al., $2010^{18}$ & 14 \\
\hline Mahure et al., $2018^{22}$ & 12 \\
\hline Nakagawa et al., $2017^{23}$ & 10 \\
\hline Porcellini et al., $2009^{10}$ & 14 \\
\hline Thal et al., $2007^{7}$ & 15 \\
\hline Van der Linde et al., $2011^{19}$ & 15 \\
\hline
\end{tabular}

difference between the mean age of males and females was statistically significant, with males being slightly younger, at a mean age of 24.6 years compared to the mean female age of 25.5 years at the time of the surgery. This study found younger age (mean age, 22.6 years), a prior closed reduction procedure, positive evidence of bilateral shoulder instability, and Caucasian ethnicity were significant risk factors for recurrence; however, they found that there was no statistically significant difference in recurrence rates between genders. ${ }^{22}$ Almeida Filho et al. ${ }^{21}$ reported the mean ages of males and females at the time of surgery to be 31.8 years and 43.5 years, respectively, and found that there was no statistically significant difference between genders with regard to recurrence.

Among the 10 studies included in this systematic review, 2 studies found a correlation between the number suture anchors and recurrence. ${ }^{17,19}$ Boileau et al. ${ }^{17}$ found that the use of "fewer than four suture anchors" intraoperatively was related to recurrence. Van der Linde et al. ${ }^{19}$ reported a similar outcome; they found an inverse relationship between the number of suture anchors used and the recurrence rate; the use of 2 sutures anchors had a higher recurrence rate than when 3 or more suture anchors were used.

\section{Clinical Outcomes}

Clinical outcomes were inconsistently reported, so no quantitative analysis of clinical outcomes or returnto-sport between sexes was possible. None of the included studies examined the difference in participation and/or return to sports between the sexes. Also, although young age (22 years or younger) was reported to be a significant risk factor in $8^{7,8,10,17,18,20,22,23}$ of the 10 studies included, the specific age breakdown of the patients who had recurrence was not reported; instead, ranges were used. No study specifically examined the age makeup of the male and female recurrences respectively. No other outcome score aside from recurrence was consistently reported and compared between the male and female cohorts.

\section{Discussion}

The principal findings of this study suggest that there is a sex-based effect that influences recurrence following arthroscopic anterior shoulder stabilization, with males having a higher incidence of recurrent instability compared to females at a minimum follow-up of 24 months. However, due to the lack of consistent reporting, this difference in recurrence that exists between the sexes cannot be attributed solely to sex itself, and no definitive conclusion can be made with the current data.

Although previous studies have identified male sex as a risk factor for recurrent shoulder instability, $8,10,24$ some studies ${ }^{5,18,21-23}$ have reported that there is no statistical difference in recurrence rates between males and females. Notably, a previous systematic review conducted by Randelli et al. ${ }^{24}$ concluded that young age, male sex and a competitive level of sports are risk factors for recurrences following arthroscopic Bankart repair. Randelli et al. ${ }^{24}$ reported a significantly higher recurrence rate in males $(15 \%)$ compared to females $(8.7 \%)$ in a 10 -year follow-up period. ${ }^{19}$ This study updates the findings in Randelli et al. ${ }^{24}$ by examining a greater number of patients but more important, it illuminates the lack of reporting that exists between the male and female cohorts. This updated systematic review found no other clinical outcome that was examined aside from recurrence that explicitly compared males and females.

Table 3. Recurrences

\begin{tabular}{|c|c|}
\hline & Recurrences \\
\hline \multicolumn{2}{|l|}{ Prospective studies $^{17}$} \\
\hline Boileau et al., $2007^{17}$ & $19 / 131(15 \%)$ \\
\hline Mahure et al., 2018 & $461 / 5719(8 \%)$ \\
\hline Porcellini et al., $2009^{10}$ & $31 / 385(8 \%)$ \\
\hline Van der Linde et al., $2011^{19}$ & $23 / 65(35 \%$ \\
\hline \multicolumn{2}{|l|}{ Retrospective studies } \\
\hline Ahmed et al., $2012^{8}$ & $40 / 302(13 \%$ \\
\hline Almeida Filho et al., $2012^{21}$ & $8 / 49(16 \%$ \\
\hline Chen et al., $2005^{20}$ & $6 / 28(21 \%$ \\
\hline Flinkkila et al., $2010^{18}$ & $33 / 174(19 \%$ \\
\hline Nakagawa et al., $2017^{23}$ & $10 / 172(6 \%)$ \\
\hline Thal et al., $2007^{7}$ & $5 / 72(35 \%$ \\
\hline
\end{tabular}




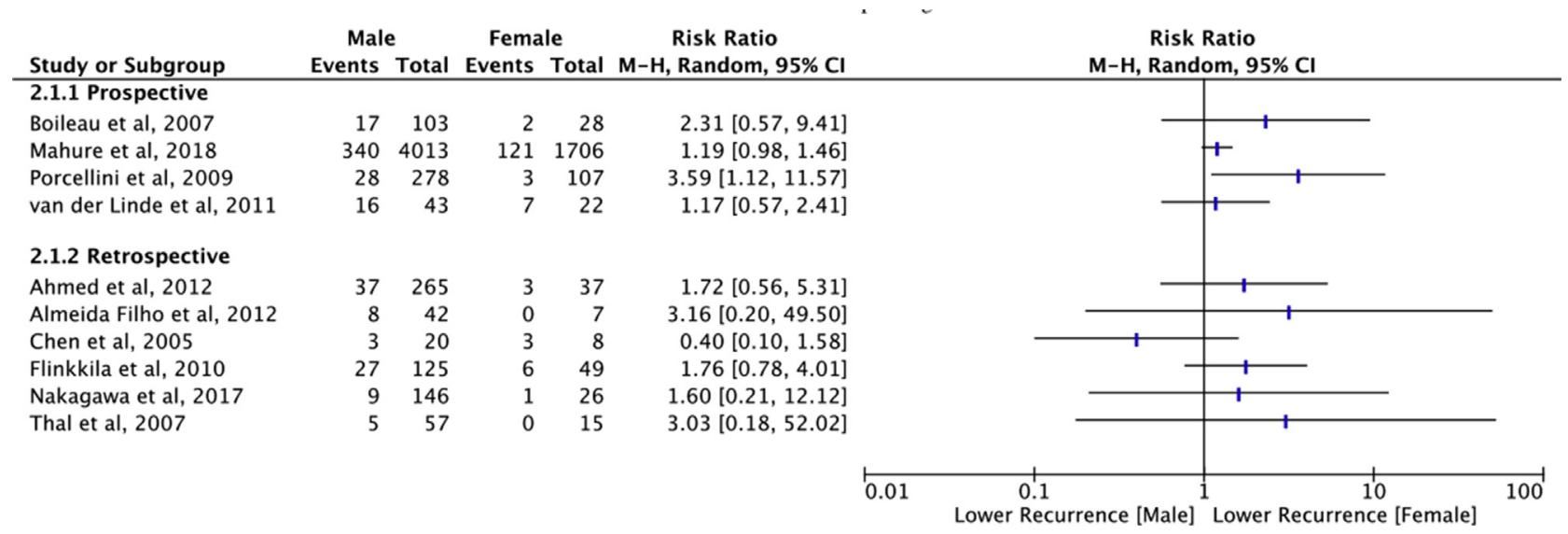

Fig 2. Forest plot showing recurrence differences between males and females following arthroscopic anterior shoulder stabilization. CI, confidence interval; $\mathrm{M}-\mathrm{H}$, Mantel-Haenszel.

Ligamentous laxity has been cited as a possible risk factor for recurrence following arthroscopic stabilization. ${ }^{17,25}$ Boileau et al. ${ }^{17}$ reported shoulder joint laxity as a significant risk factor for recurrence following arthroscopic stabilization. They developed a 10-point instability severity index score, which included shoulder joint laxity, as simple preoperative tool to assess patients' risk of developing recurrent instability following arthroscopic stabilization of anterior shoulder instability. ${ }^{17}$ In this study, they found that an instability severity index score of greater than 6 conferred a $70 \%$ risk of recurrence following arthroscopic stabilization. ${ }^{17}$

However, although both of these studies ${ }^{17,25}$ did examine the factor of laxity, they did not evaluate joint laxity in regard to males and females independently. Joint laxity is of particular interest because it has been reported that females have a higher rate of joint laxity. ${ }^{26}$ Reuter et al. ${ }^{26}$ observed females to have a statistically significant higher Beighton Hypermobility Score, ${ }^{27}$ which correlates with increased joint laxity, than the Beighton Hypermobility Score scores of males included in the study. ${ }^{26}$ However, this significantly higher difference in general joint hyperlaxity in females did not correlate with a higher rate of shoulder instability in females than in males. This conclusion is interesting because it possibly implies that although joint laxity is a significant risk factor for shoulder instability, the effect may be felt differently in males and females. In regard to recurrence, joint laxity may influence males' outcomes more than females'; however, with the current lack of data reported for males and females independently, this is unable to be investigated.

Sports-related instability was also reported to be higher among males by Wagstrom et al. ${ }^{3}$ in their study that examined glenohumeral instability in athletes. The data concluded that there was a 4.0 to 1.0 maleto-female ratio in regard to experiencing glenohumeral instability. ${ }^{3}$ The most common sports in which glenohumeral instability was seen were football, wrestling and basketball, and it was reported that nearly all cases were the result of trauma. ${ }^{3}$ The findings that males are more likely to sustain recurrence due to sport is interesting when looking at the time it takes males and females to return to sport. A previous study looking at return to sport following arthroscopic Bankart stabilization concluded that male athletes returned to preoperative sports levels significantly sooner $(P<0.001)$ than female athletes. ${ }^{28}$ This faster return to preoperative levels of sports in males could be a possible explanation for the reason males are at a higher risk of recurrence following arthroscopic stabilization. One possible explanation could be that male athletes feel more confident more quickly than females following arthroscopic repair and do not allow sufficient time for healing. A recent study by Webster et al. ${ }^{29}$ found that male patients had a higher psychological readiness to return to sport following anterior cruciate ligament reconstruction when compared to females. It is possible that similar findings following anterior shoulder stabilization procedures may contribute to the increased recurrence rates in males.

\section{Limitations}

The limitations of this study should also be considered. Of the 10 studies included, 5 studies were Level III and 3 were Level IV. Based on the risk of bias analysis using the MINORS score, 5 studies had a relatively low score (ranging from 10 to 13 ), 8,12,20,22,23 which indicated the possibility of a higher risk of bias. Taking this bias calculation into account and the fact that the included studies are composed mostly of higher-level studies (Level III and IV), the pooling of data and weighted mean calculations were avoided. Although the MCMS methodology score and the MINORS score for risk of bias were employed to ensure the quality of the included studies, it recognized that these scores tend to overestimate the strengthens of the included studies 
due to the pooling of individual scores and the reporting of the average. ${ }^{30}$ It is also of note that $81 \%$ of the data came from 1 study by Mahure et al. ${ }^{22}$; therefore, the results may have been significantly influenced by this study. However, if the data from Mahure et al. ${ }^{22}$ were not included, a similar difference in recurrences would still exist between males and females in the remaining patient population. No other clinical outcome, aside from recurrence, was available for comparison between male and female patients. Of the 10 studies included in this systematic review, only 3 clearly identified the age makeup of the male and female patients who were included. However, the specific ages of the males and females who experienced recurrence were not reported. It has been previously reported ${ }^{10,31}$ that younger age is a significant risk factor for recurrence. So, without the complete age makeup of the male and female patient populations, no relationship among gender, age and recurrence could be inferred. ${ }^{10,11}$ Therefore, it cannot be ruled out that the lower recurrence rate that is seen in the female cohort is not due to the possible older age makeup of the female cohort. Also, with regard to age, there was a wide age range of patients included in this study, ranging from 14 to 69 years of age at the time of surgery. This wide range and the fact that different age cohorts inherently carry totally different levels of risk should be taken into account. Among the included studies, there was also a wide range in follow-up duration (24 months to 120 months), which likely influenced the differences in recurrence rates that were reported among the studies. Longer follow-up periods inherently carry an increased risk of recurrence. Because of the nature of this systematic review and the lack of consistent reporting by the included studies, confounding variables, such as laxity, physical activity, return to sport, and return at how long after surgery, could not be controlled.

\section{Conclusions}

Following arthroscopic anterior shoulder stabilization, males have a higher recurrence rate following arthroscopic anterior shoulder stabilization than females. Future studies should aim to determine a possible explanation for the higher rate of recurrent instability in male patients compared to female patients in order to reduce recurrence rates following surgery.

\section{References}

1. Szyluk $\mathrm{K}$, Jasinski A, Niemiec $\mathrm{P}$, Mielnik M, Widuchowski W, Koczy B. Male gender and age range 2029 years are the most important non-modifiable risk factors for recurrence after primary post-traumatic shoulder dislocation. Knee Surg Sports Traumatol Arthrosc 2018;26:2454-2464.
2. Kraeutler MJ, Currie DW, Kerr ZY, Roos KG, McCarty EC, Comstock RD. Epidemiology of shoulder dislocations in high school and collegiate athletics in the United States: 2004/2005 through 2013/2014. Sports Health 2018;10: 85-91.

3. Wagstrom E, Raynor B, Jani S, et al. Epidemiology of Glenohumeral instability related to sporting activities using the FEDS (Frequency, Etiology, Direction, and Severity) classification system: A multicenter analysis. Orthop J Sports Med 2019;7:2325967119861038.

4. Brophy RH, Hettrich CM, Ortiz S, Group MSI, Wolf BR. Patients undergoing shoulder stabilization surgery have elevated shoulder activity compared with sex- and agematched healthy controls. Sports Health 2016;9:59-63.

5. Aboalata M, Plath JE, Seppel G, Juretzko J, Vogt S, Imhoff $\mathrm{AB}$. Results of arthroscopic Bankart repair for anterior-inferior shoulder instability at 13-year follow-up. Am J Sports Med 2017;45:782-787.

6. Aboalata M, Halawa A, Basyoni Y. The double Bankart bridge: A technique for restoration of the labral footprint in arthroscopic shoulder instability Repair. Arthrosc Tech 2017;6:e43-e47.

7. Thal R, Nofziger M, Bridges M, Kim JJ. Arthroscopic Bankart repair using knotless or BioKnotless suture anchors: 2- to 7-year results. Arthroscopy 2007;23: 367-375.

8. Ahmed I, Ashton F, Robinson CM. Arthroscopic Bankart repair and capsular shift for recurrent anterior shoulder instability: Functional outcomes and identification of risk factors for recurrence. J Bone Joint Surg Am 2012;94: 1308-1315.

9. Zimmermann SM, Scheyerer MJ, Farshad M, Catanzaro S, Rahm S, Gerber C. Long-term restoration of anterior shoulder stability: A retrospective analysis of arthroscopic Bankart repair versus open latarjet procedure. J Bone Joint Surg Am 2016;98:1954-1961.

10. Porcellini G, Campi F, Pegreffi F, Castagna A, Paladini P. Predisposing factors for recurrent shoulder dislocation after arthroscopic treatment. J Bone Joint Surg Am 2009;91: 2537-2542.

11. Kitagawa T, Matsui N, Nakaizumi D. Impact of combination of therapeutic exercise and psychological intervention for a patient with first-time traumatic shoulder dislocation. J Phys Ther Sci 2019;31:850-854.

12. de Almeida Filho IA, de Castro Veado MA, Fim M, da Silva Corrêa LV, de Carvalho Junior AER. Functional assessment of arthroscopic repair for recurrent anterior shoulder instability. Revista Brasileira de Ortopedia (Eng. Ed.) 2012;47:214-221.

13. Coleman BD, Khan KM, Maffulli N, Cook JL, Wark JD. Studies of surgical outcome after patellar tendinopathy: clinical significance of methodological deficiencies and guidelines for future studies. Victorian Institute of Sport Tendon Study Group. Scand J Med Sci Sports 2000;10: 2-11.

14. Floyd EM, Ho S, Patel P, Rosenfeld RM, Gordin E. Systematic review and meta-analysis of studies evaluating functional rhinoplasty outcomes with the NOSE Score. Otolaryngol Head Neck Surg 2017;156:809-815.

15. Cote MP, Lubowitz JH, Rossi MJ, Brand JC. Reviews pooling heterogeneous, low-evidence, high-bias data 
result in incorrect conclusions: But heterogeneity is an opportunity to explore. Arthroscopy 2018;34:3126-3128.

16. Version 5.3. Copenhagen. The Nordic Cochrane Centre: The Cochrane Collaboration; 2014.

17. Balg F, Boileau P. The instability severity index score. A simple pre-operative score to select patients for arthroscopic or open shoulder stabilisation. J Bone Joint Surg Br 2007;89:1470- 1477 .

18. Flinkkila T, Hyvonen P, Ohtonen P, Leppilahti J. Arthroscopic Bankart repair: Results and risk factors of recurrence of instability. Knee Surg Sports Traumatol Arthrose 2010;18:1752-1758.

19. van der Linde JA, van Kampen DA, Terwee CB, Dijksman LM, Kleinjan G, Willems WJ. Long-term results after arthroscopic shoulder stabilization using suture anchors: An 8- to 10-year follow-up. Am J Sports Med $2011 ; 39: 2396-2403$.

20. Chen S, Haen PS, Walton J, Murrell GA. The effects of thermal capsular shrinkage on the outcomes of arthroscopic stabilization for primary anterior shoulder instability. Am J Sports Med 2005;33:705-711.

21. de Almeida Filho IA, de Castro Veado MA, Fim M, da Silva Correa LV, de Carvalho Junior AE. Functional assessment of arthroscopic repair for recurrent anterior shoulder instability. Rev Bras Ortop 2012;47:214-221.

22. Mahure SA, Mollon B, Capogna BM, Zuckerman JD, Kwon YW, Rokito AS. Risk factors for recurrent instability or revision surgery following arthroscopic Bankart repair. Bone Joint J 2018;100-B:324-330.

23. Nakagawa S, Iuchi R, Mae T, Mizuno N, Take Y. Clinical outcome of arthroscopic Bankart repair combined with simultaneous capsular repair. Am J Sports Med 2017;45: 1289-1296.

24. Randelli P, Ragone V, Carminati S, Cabitza P. Risk factors for recurrence after Bankart repair: A systematic review. Knee Surg Sports Traumatol Arthrosc 2012;20:2129-2138.

25. Kasik CS, Rosen MR, Saper MG, Zondervan RL. High rate of return to sport in adolescent athletes following anterior shoulder stabilisation: A systematic review. J ISAKOS 2019;4:33-40.

26. Reuter PR, Fichthorn KR. Prevalence of generalized joint hypermobility, musculoskeletal injuries, and chronic musculoskeletal pain among American university students. PeerJ 2019;7:e7625.

27. Beighton P, Horan F. Orthopaedic aspects of the EhlersDanlos syndrome. J Bone Joint Surg Br 1969;51:444-453.

28. Gerometta A, Rosso C, Klouche S, Hardy P. Arthroscopic Bankart shoulder stabilization in athletes: Return to sports and functional outcomes. Knee Surg Sports Traumatol Arthrosc 2016;24:1877-1883.

29. Webster KE, Nagelli CV, Hewett TE, Feller JA. Factors associated with psychological readiness to return to sport after anterior cruciate ligament reconstruction surgery. Am J Sports Med 2018;46:1545-1550.

30. Greenland S, O'Rourke K. On the bias produced by quality scores in meta-analysis, and a hierarchical view of proposed solutions. Biostatistics 2001;2:463-471.

31. Olds M, Ellis R, Donaldson K, Parmar P, Kersten P. Risk factors which predispose first-time traumatic anterior shoulder dislocations to recurrent instability in adults: A systematic review and meta-analysis. Br J Sports Med 2015:49:913-922. 